\title{
Dynamic and Static Water Molecules Complement the TN16 Conformational Heterogeneity Inside the Tubulin Cavity
}

\author{
Sarmistha Majumdar, Satyabrata Maiti, Shubhra Ghosh Dastidar* \\ Bioinformatics Centre, Bose Institute, P-1/12 CIT Scheme VII M, Kolkata 700054
}

\section{Supporting Information}

Further details on the structure and dynamics: A glimpse of the dynamics of the TN16 has been shown in a movie file (M1.avi) which demonstrates the entry of water molecules into the binding pocket and the flip of the ligand in the TBE200I1 trajectory. The water around the ligand within the binding pocket of the tubulin are also mobile. Although they are often forming bridging interactions, but they also leave the binding pocket and get replaced by a newer molecule. On an average the total number of water molecules present within the binding pocket remains constant and their sites remains preserved. The Table S1 presents a list of such sites and the lifetime of H-bond interactions: (i) with TN16 and with $\beta$-subunit of the protein separately for the complexes and (ii) with $\beta$-subunit of the protein only for the complexed cases. The conformational preference for the TN16 in its uncomplexed state (TN16U) and for the complexed states (TBE200I1, TBE200I2, TBE200P) have been shown in Fig S2. It shows that the conformations sampled in the TBE200I1 due to flipping of TN16 is also sampled by the uncomplexed TN16 although with lower priority, which indicates that interactions from the surroundings are required to stabilize this flipped conformation. The Fig S3-6 present two dimensional representation of the tubulin-TN16 interactions in a comprehensive way.

Movie M1: A separately enclosed movie file M1.avi shows a selected portion of the TBE200I1 trajectory the details of which has been presented in the main text. 
Table S1: H-bond life time and average no of water molecules that visit the ligand binding pocket

\begin{tabular}{|c|c|c|c|c|}
\hline \multirow[t]{2}{*}{ System name } & \multirow{2}{*}{$\begin{array}{l}\text { Water molecules within the } \\
\text { TN16-binding pocket of } \\
\text { tubulin protein forming } \\
\text { Hbonds }\end{array}$} & \multicolumn{2}{|c|}{$\begin{array}{l}\text { Average } \mathrm{H} \text {-bond lifetime } \\
\text { (ps) for water }\end{array}$} & \multirow{2}{*}{$\begin{array}{l}\text { Average no. of } \\
\text { binding pocket } \\
\text { visiting water } \\
\text { molecules }\end{array}$} \\
\hline & & $\begin{array}{l}\text { With } \\
\text { TN16 }\end{array}$ & $\begin{array}{l}\text { With } \beta- \\
\text { subunit of } \\
\text { the protein }\end{array}$ & \\
\hline TBE200I1 & $\begin{array}{l}\text { wat1 } \\
\text { wat2 } \\
\text { wat3 } \\
\text { wat4 } \\
\text { wat5 } \\
\text { wat6 }\end{array}$ & $\begin{array}{r}744.0 \\
13.6 \\
15.1 \\
13.6 \\
7.0 \\
9.6\end{array}$ & $\begin{array}{r}135.4 \\
85.6 \\
54.7 \\
25.0 \\
43.3 \\
18.5\end{array}$ & 4 \\
\hline TBE200I2 & $\begin{array}{l}\text { wat1 } \\
\text { wat2 } \\
\text { wat3 }\end{array}$ & $\begin{array}{l}83.5 \\
21.7 \\
19.7\end{array}$ & $\begin{array}{r}102.5 \\
87.3 \\
33.9\end{array}$ & 9 \\
\hline TBE200P & wat1 & 16.5 & 63.4 & 6 \\
\hline TBUC & $\begin{array}{l}\text { wat1 } \\
\text { wat2 } \\
\text { wat3 } \\
\text { wat4 } \\
\text { wat5 } \\
\text { wat6 } \\
\text { wat7 } \\
\text { wat8 } \\
\text { wat9 } \\
\text { wat10 } \\
\text { wat11 }\end{array}$ & & $\begin{array}{r}10.1 \\
11.2 \\
7.1 \\
12.0 \\
9.3 \\
8.4 \\
23.6 \\
126.3 \\
10.2 \\
8.2 \\
9.3\end{array}$ & 14 \\
\hline TUBS & $\begin{array}{l}\text { wat1 } \\
\text { wat2 } \\
\text { wat3 } \\
\text { wat4 } \\
\text { wat5 } \\
\text { wat6 }\end{array}$ & & $\begin{array}{r}8.3 \\
121.1 \\
18.3 \\
11.2 \\
13.3 \\
8.8\end{array}$ & 9 \\
\hline
\end{tabular}




\section{Figures:}

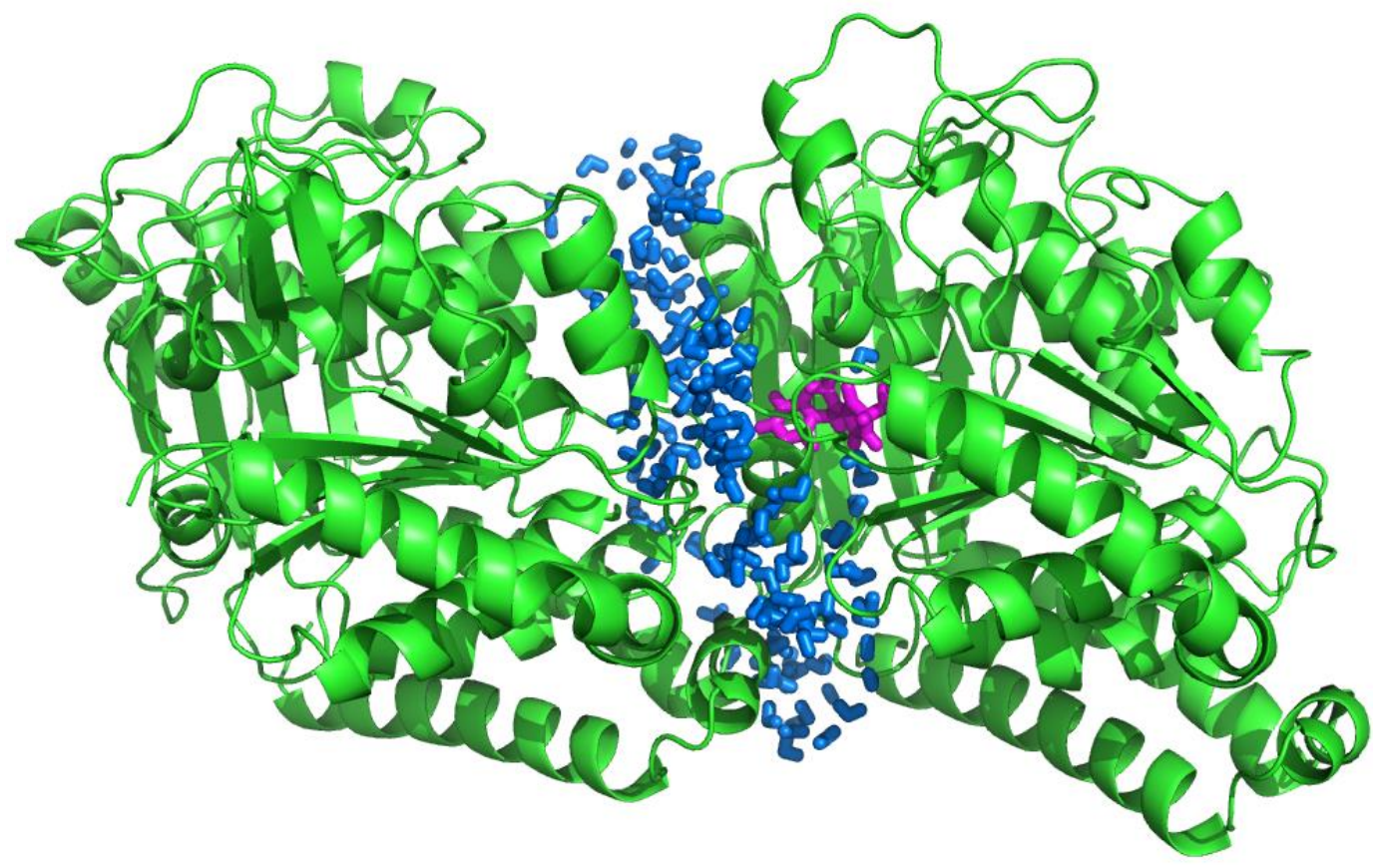

Figure S1: A thin film of water in between the space of $\alpha$ and $\beta$ subunit of tubulin was represented in blue and ligand TN16 was shown in pink 

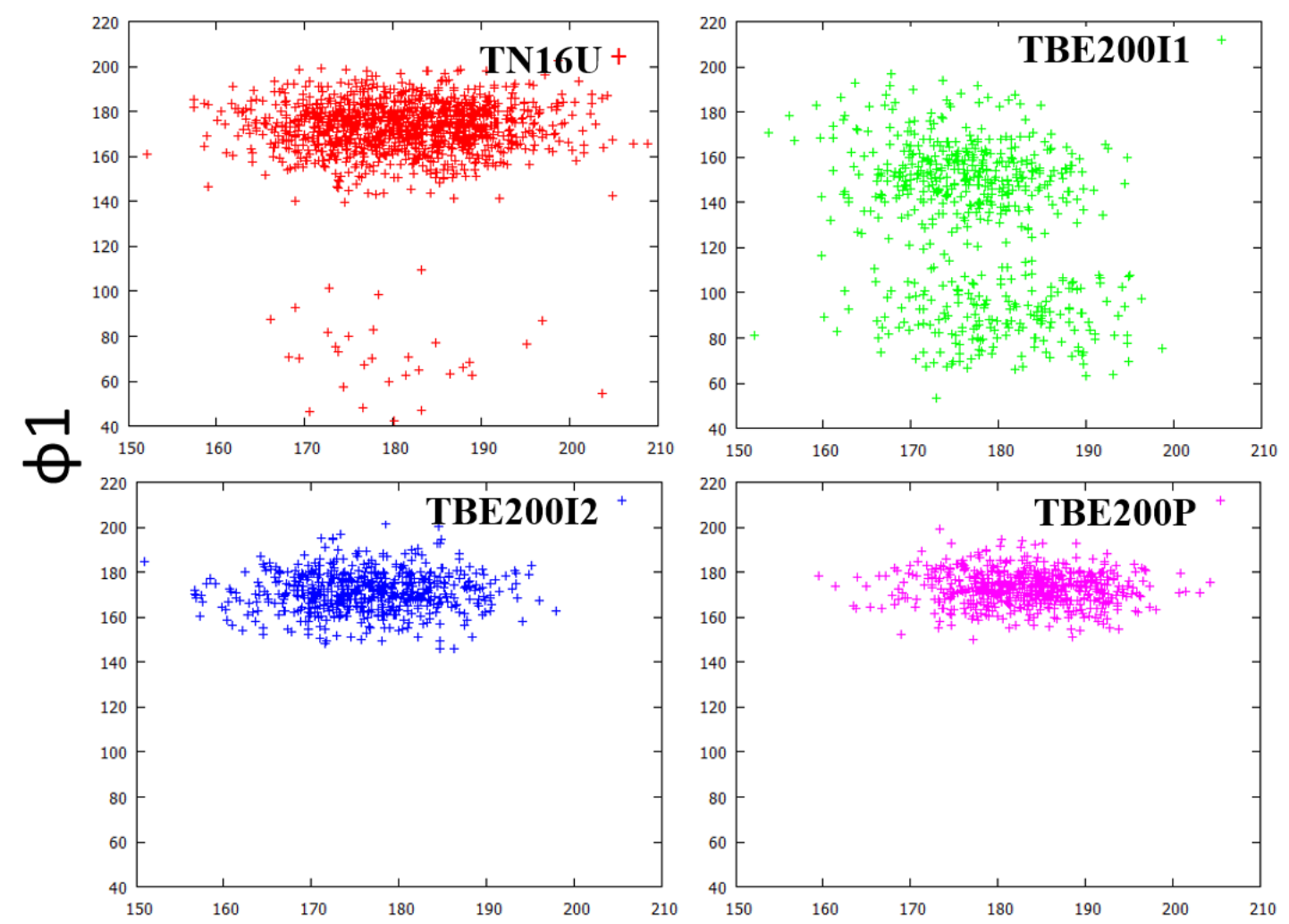

$\phi 2$

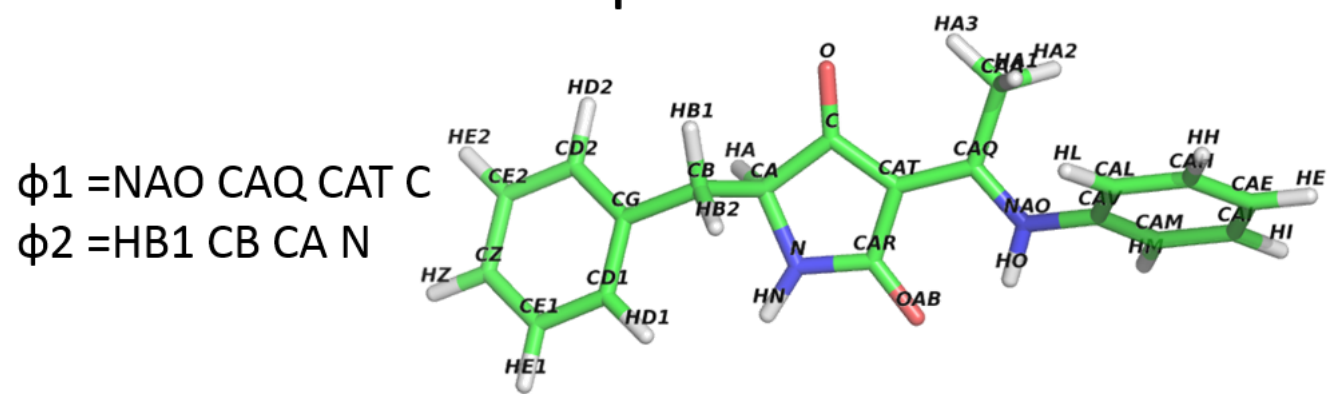

Figure S2: Sampled conformations of TN16 in terms of two dihedral angles $\Phi 1$ and $\Phi 2$ of uncomplexed and complexed systems. System specifications are given in the right corner of each 


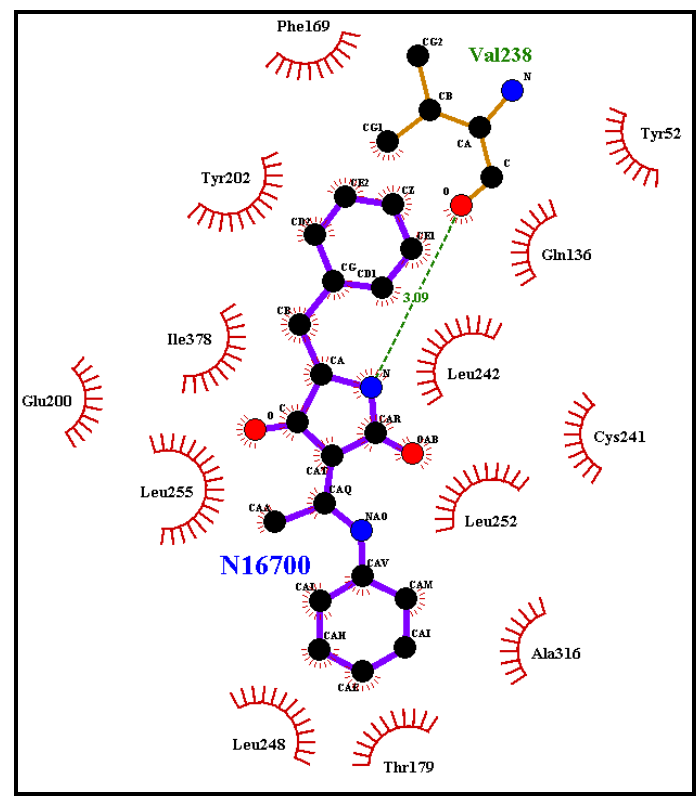

Figure S3: Ligand protein interactions as observed in crystal structure (PDB ID-3HKD) using Ligplot: TN16 (N16700) makes hydrogen bond with V238 of tubulin protein chain B and also form hydrophobic interactions with many surrounding amino acid residues which are represented in red 'semi-circle'.

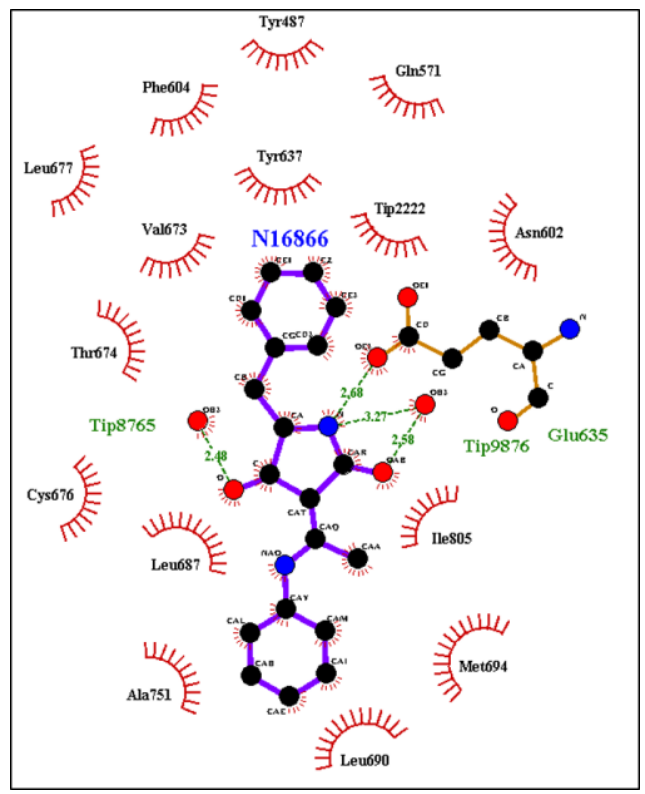

Figure S4: Ligand protein interactions observed in "TBE200I1" using ligplot: TN16 (N16866) has polar interaction with E635(200) through hydrogen bond formation. The numbers within the bracket ' () ' represent the residue id of the respective amino acid residues as per crystal structure. Additionally, two water molecules (Tip9876, Tip8765) are also involved in polar interactions with ligand and few amino acids via hydrogen bonds. 


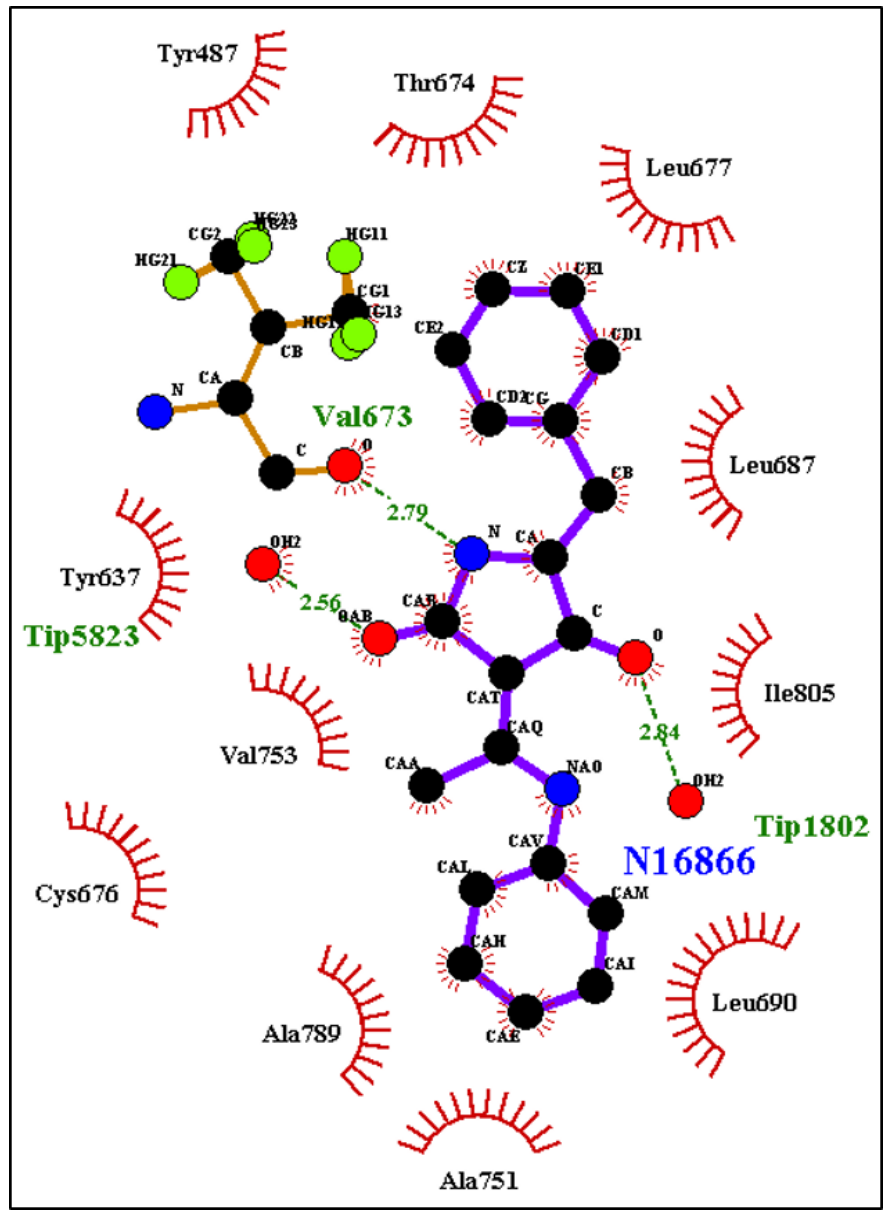

Figure S5: Ligand protein interactions observed in "TBE200I2" using ligplot: : TN16 (N16866) interacts with V673(238) through hydrogen bond formation. Two coodinating water molecules (Tip5823, Tip1802) also facilitate the binding of TN16(N16866) by forming hydrogen bonds. 


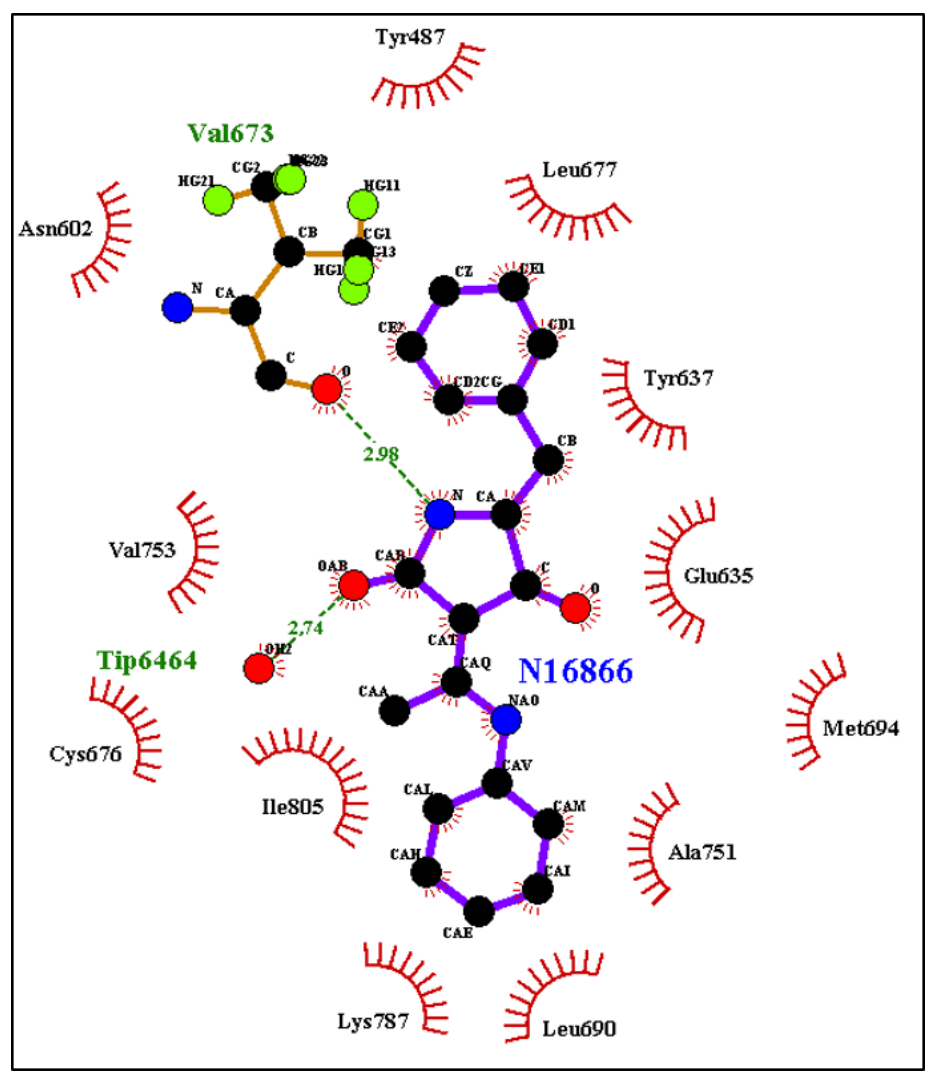

Figure S6: Ligand protein interactions observed in "TBE200P" using ligplot: TN16 (N16866) interacts with V673(238) through hydrogen bond formation and also interacts with a water molecule (Tip6464) inside the binding pocket.

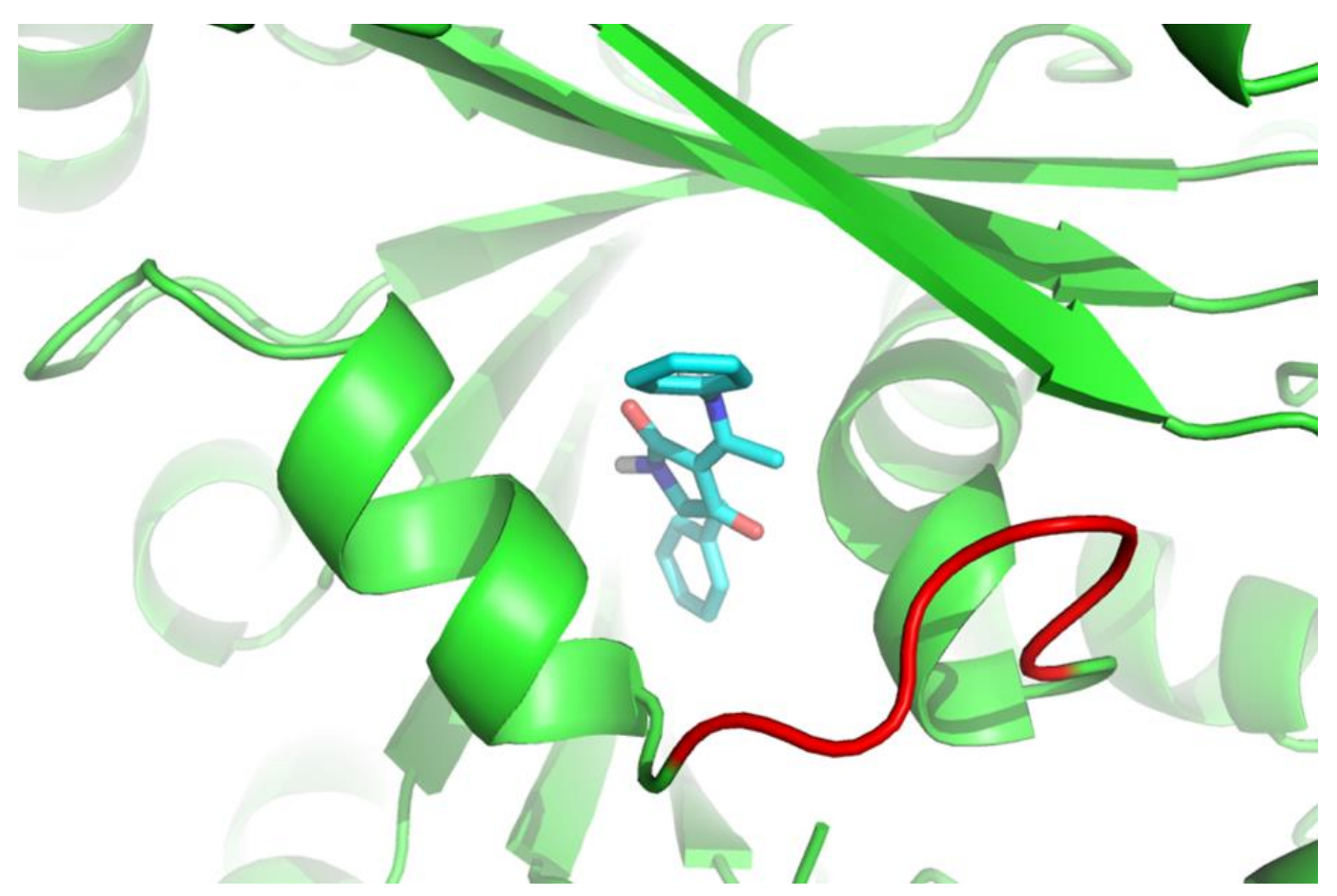

Figure S7: Location of TN16 ligand within the $\beta$ subunit of tubulin protein where the T7 loop was colored in red. 


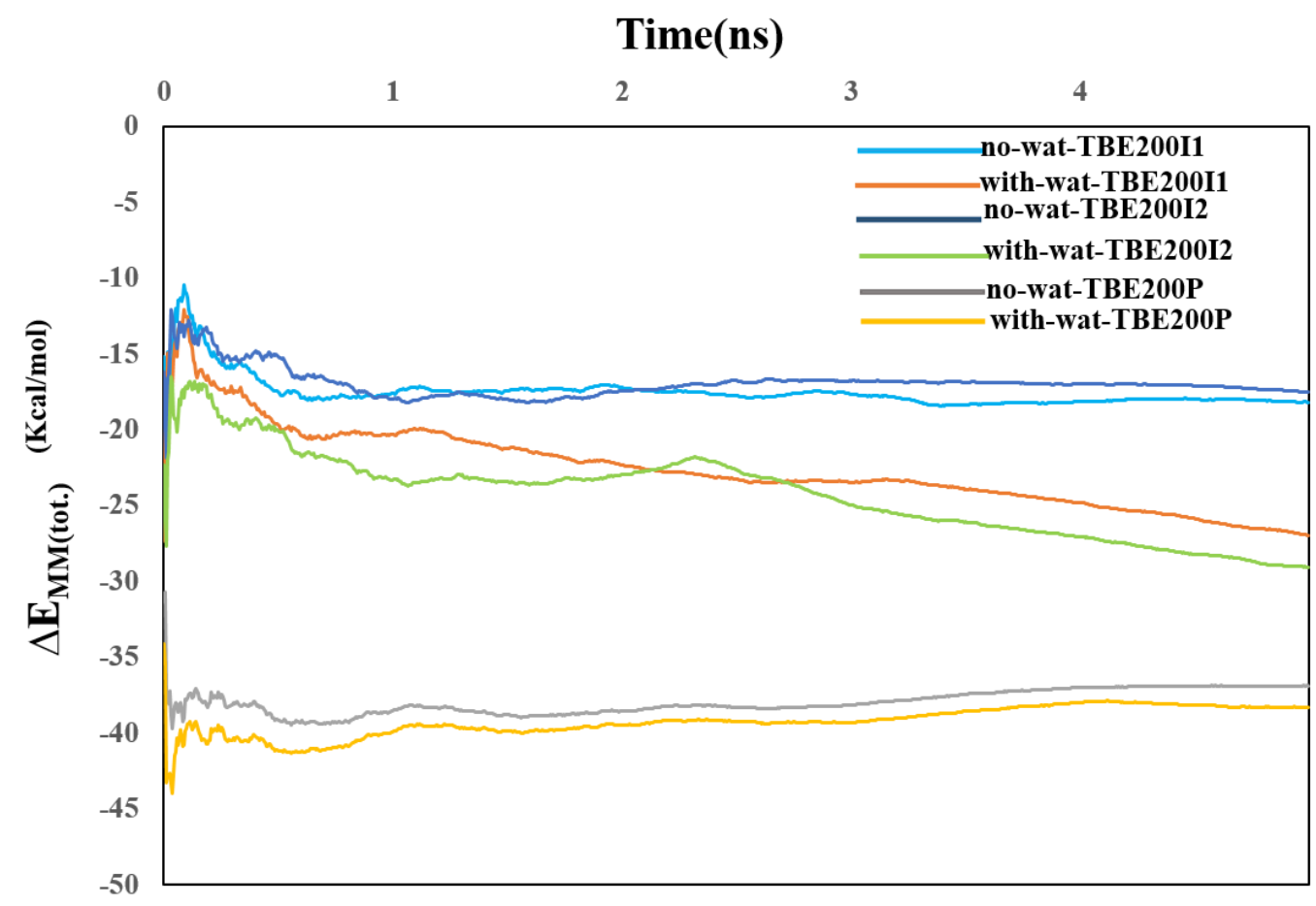

Figure S8: Cumulative average of the binding energy as a function of first 5 ns of simulation length. 